\title{
A Non-ischemic Spontaneous Papillary Muscle Rupture. Case Report
}

\author{
Erik Orozco-Hernandez ${ }^{1}$, Heriberto Matos-Santana ${ }^{1}$, Jose Tallaj ${ }^{2}$, Lluz Padilla ${ }^{3}$, and James \\ Davies $^{4}$
}

${ }^{1}$ The University of Alabama at Birmingham School of Medicine

${ }^{2}$ The University of Alabama at Birmingham Department of Surgery

${ }^{3}$ The University of Alabama at Birmingham

${ }^{4}$ University of Alabama at Birmingham

February 9, 2021

\begin{abstract}
We report a case of spontaneous mechanical papillary muscle rupture (PMR). We believe that the etiology of PMR, in this case, is related to a histological damage of the valve, extreme sudden force to the papillary muscle, and some possible tissue hypo-perfusion of the subvalvular apparatus. We performed a mitral valve replacement and bypass coronary surgery with good results.
\end{abstract}

\section{Introduction}

Papillary muscle rupture (PMR) is one of the most serious mechanical complications of acute myocardial infarction, with an incidence of 0.5-5\% (1). Some reports of PMR not related with ischemic failure include idiopathic, infectious or connective tissue disorder etiologies (2), including Takotsubo disease $(3,4)$. In this paper, we present a case of non-ischemic spontaneous PMR, potentially related with extreme physical activity.

\section{Case report:}

This report was waived from institutional review board approval as it does not constitute human subjects research, consent was not obtained as this case contains no personal health identifiers. A 59-year-old male with no medical history developed sudden onset of dyspnea shortly after strenuous physical activity loading a truck with a couch. He presented to a local community hospital but during his transport he was drowsy and required bag valve mask ventilation. On arrival to the emergency department his telemetry showed atrial fibrillation with rapid ventricular response and no ST-segment changes. The patient underwent endotracheal intubation due to respiratory distress secondary to pulmonary edema. Bedside echocardiogram was performed, showing severe mitral valve regurgitation suspect of anterior chordae leaflet rupture. An intraortic balloon pump was placed for support in addition to intravenous vasopressors (epinephrine and vasopressin). He was transferred to a tertiary center for urgent valve repair.

Arrival laboratory workup demonstrated a serum creatinine of $2.3 \mathrm{mg} / \mathrm{dl}$, hemoglobin $10.8 \mathrm{~g} / \mathrm{L}$, platelets $242 \mathrm{x}$ $10^{\wedge} 3 / \mathrm{cmm}$, white blood cell count $1710^{\wedge} 3 / \mathrm{cmm}$, and a peak troponin-I of $0.490 \mathrm{ng} / \mathrm{mL}$. He was started on broad spectrum antibiotics for suspected aspiration pneumonia on chest x-ray. A transthoracic echocardiogram showed anterior mitral valve leaflet flail suspecting ruptured chordae, normal biventricular function, and no regional wall motions abnormalities. A cardiac catheterization was performed, demonstrating 40-50\% ostial left main disease with mild-moderate disease in the rest of the coronary arteries providing TIMI III 
flow. A transesophageal echocardiography added supportive evidence of a ruptured chord leading to A1 flail and severe mitral regurgitation (Figure1 A and 1C). The regurgitant jet was posteriorly directed and exhibited a Coanda effect (Figure 1B). The EROA by PISA was $0.79 \mathrm{~cm} \backslash S \backslash 2, \mathrm{VC}$ was $0.80 \mathrm{~cm}$ and regurgitant volume was $73 \mathrm{~mL}$. EROA by $3 \mathrm{D}$ was $1.10 \mathrm{~cm} \backslash S \backslash 2$. There was evidence of reverse systolic flow in the pulmonary veins with severe mitral regurgitation. Left ventricular ejection fraction was described as normal. The patient underwent mitral valve replacement with a bioprosthetic valve and bypass grafting of two coronary vessels. There was no macroscopic evidence of acute ischemia or myocardial infarction, the coronary vessels showed mild eccentric atheromatosis. The excision of the mitral valve revealed the anterolateral PMR attached to the apparatus (Figure 1D). Pathology demonstrated a valve tissue with fibrosis and myxoid degeneration. The hospital course was complicated with self-limited gastrointestinal bleed, new onset atrial fibrillation, and a small thromboembolic cerebrovascular accident. The patient ultimately recovered and was discharged home with a NYHA I functional class and a transthoracic echocardiogram showing normal biventricular function and a bioprostethic mitral valve with normal gradients and no perivalvular leaks.

\section{Discussion}

PMR is a rare but life-threatening emergency, generally associated with coronary artery disease, specifically acute myocardial ischemia. There have been other non-ischemic etiologies related to PMR. Gouda et al published a review of all non-ischemic spontaneous PMR case reports up to 2015 (2), differential etiologies noted in the summary include myocarditis $(5,6)$, endocarditis and fungal infections $(7,8)$, Ehler Danlos type IV, Takotsubo cardiomyopathy and mitral annular calcification are other described entities $(3,4,9,10)$. From the 12 cases published thus far, 4 of them had an unknown etiology $(2,11,12,13)$.

In our case, the angiography showed some coronary artery disease, but nothing supported an acute coronary syndrome. Furthermore, there was no previous clinical evidence of stable angina, abnormal wall motion, recent infarct, hypertension, vasculitis, trauma, hypercoagulable state, infection and no clinical and/or surgical evidence of ventricular hypertrophy that could justify the rupture.

We believe three things could explain the spontaneous PMR in our patient, one is related with the pathology findings of fibrosis and myxoid degeneration in the mitral valve, Seve and Shim $(9,10)$ published connective tissue disorders (Ehler Danlos) as a cause for rupture. The second important factor in our patient is the history of sudden onset of dyspnea shortly after strenuous physical activity. The papillary muscle works as an independent mechanism to absorb and compensate sudden geometric changes of the left ventricular wall and to maintain the basic mitral valve geometry (14). Blunt trauma itself explains how an extreme and critical change of the intrathoracic pressure can cause rupture of the papillary muscle (2), through probable failure of the mitral subvalvular apparatus in maintaining the ventricular geometry. The Valsalva respiratory phenomenon associated with extreme exercise (as lifting a heavy weight) could be analogous to the blunt chest trauma with a closed glottis.

And finally, even when this patient did not have an acute coronary syndrome or previous myocardial infarction, we believe that there may have been possible hypoperfusion from microvascular disease or existing coronary artery disease, which could have been an added factor to this complication (2). Mitral surgery is mandatory and urgent once a PMR diagnosis is made, the standard treatment is valve replacement, although, Fiore et al., reported a case of a successful mitral valve repair with papillary muscle reimplantation (14).

The presence or combination of a tissue myxoid degeneration in the mitral valve, with an extreme force applied suddenly in the chordae tendinea and papillary muscle, and some possible tissue hypoperfusion of the subvalvular apparatus, should be considered etiologies in spontaneous PMR.

\section{References}

1. Sanders RJ, Neubuerger KT, Ravin ABE. Rupture of papillary muscles: occurrence of rupture of the posterior muscle in posterior myocardial infarction. Chest 1957;31:316-323

2. Gouda P, Weilovitch L, Kanani R, Har B. Case report and review of non-ischemic spontaneous papillary muscle rupture reports between 2000 and 2015. Echocardiography 2017; 34:786-90. 
3. Yaghoubi AR, Ansarin K, Hashemzadeh S, Azhough R, Faraji S, Bozorgi F. Tako-tsubo cardiomyopathy induced by emotional stress leading to severe mitral regurgitation, cardiogenic shock and cardiopulmonary arrest. Int J Cardiol 2009;135:e85-e86.

4. Nef HM, Möllmann H, Hilpert P, et al. Severe mitral regurgitation in Tako-Tsubo cardiomyopathy. Int J Cardiol 2009; 132:e77-e79.

5. Maruo T, Komiya T, Shimamoto T, Kadota K, Mitsudo K. Spontaneous papillary muscle rupture with localized endocarditis. Eur Heart J Cardiovasc Imaging 2015;16:115.

6. Voros G, Ishaq M, Dens J. Spontaneous rupture of a papillary muscle in acute myocarditis. Acta Cardiologica 2011;66:405-406.

7. Ahn YH, Kim NH, Song MJ, et al. Papillary muscle rupture complicating a papillary muscle abscess. Korean Circ J 2006;36:242-244.

8. Kotanidou AN, Zakynthinos E, Andrianakis I, et al. Aspergillusendocarditis in a native valve after amphotericin B treatment. Ann Thorac Surg 2004;78:1453-1455

9. Sève P, Dubreuil O, Farhat F, Plauchu H, Touboul P, Broussolle C. Acute mitral regurgitation caused by papillary muscle rupture in the immediate postpartum period revealing Ehlers-Danlos

Syndrome type IV. J Thorac Cardiovasc Surg 2005;129:680-681.

10. Shim CY, Kim YJ, Son JW, et al. Spontaneous rupture of a papillary muscle. Circulation 2013;127:e586e 588

11. Lazar HL, Bernard SA. Acute anterolateral papillary muscle rupture in the absence of coronary artery disease. J Card Surg 2010;25:518-9.

12. Lee H, Ruzsics B, Schoepf UJ, et al. New-onset heart failure caused by spontaneous papillary muscle rupture: diagnosis with dual-source computed tomographic coronary angiography. J Thorac Cardiovasc Surg 2009;137:e19-e21.

13. Hansen AJ, Sorrell VL, Cooper AD, Moulton MJ. Postpartum rupture of the posteromedial papillary muscle. J Card Surg 2012;27: 313-316.

14. Fiore A, Piscitelli M, Bergoend E, Couetil JP. Spontaneous mechanical rupture of a papillary muscle effectively resolved by mitral valve repair . European Jou of Cardiothorac Surg Volume 53, Issue 4, April 2018, Pages 874-876

Figure 1 Legend

Figure A: Transesophageal echocardiogram demonstrating a mid-esophageal 4-chamber view with the anterior leaflet of the mitral valve prolapsing into the left atrium on diastole.

Figure B: Transesophageal echocardiogram image with torrential eccentric posterior regurgitant jet of the mitral valve creating a "Coanda effect".

Figure C: Transesophageal echocardiogram with an echo dense structure at the end of the anterior leaflet representative of ruptured papillary muscle.

Figure D: Excised anterior leaflet of mitral valve, showing the rupture in the head of the papillary muscle. 


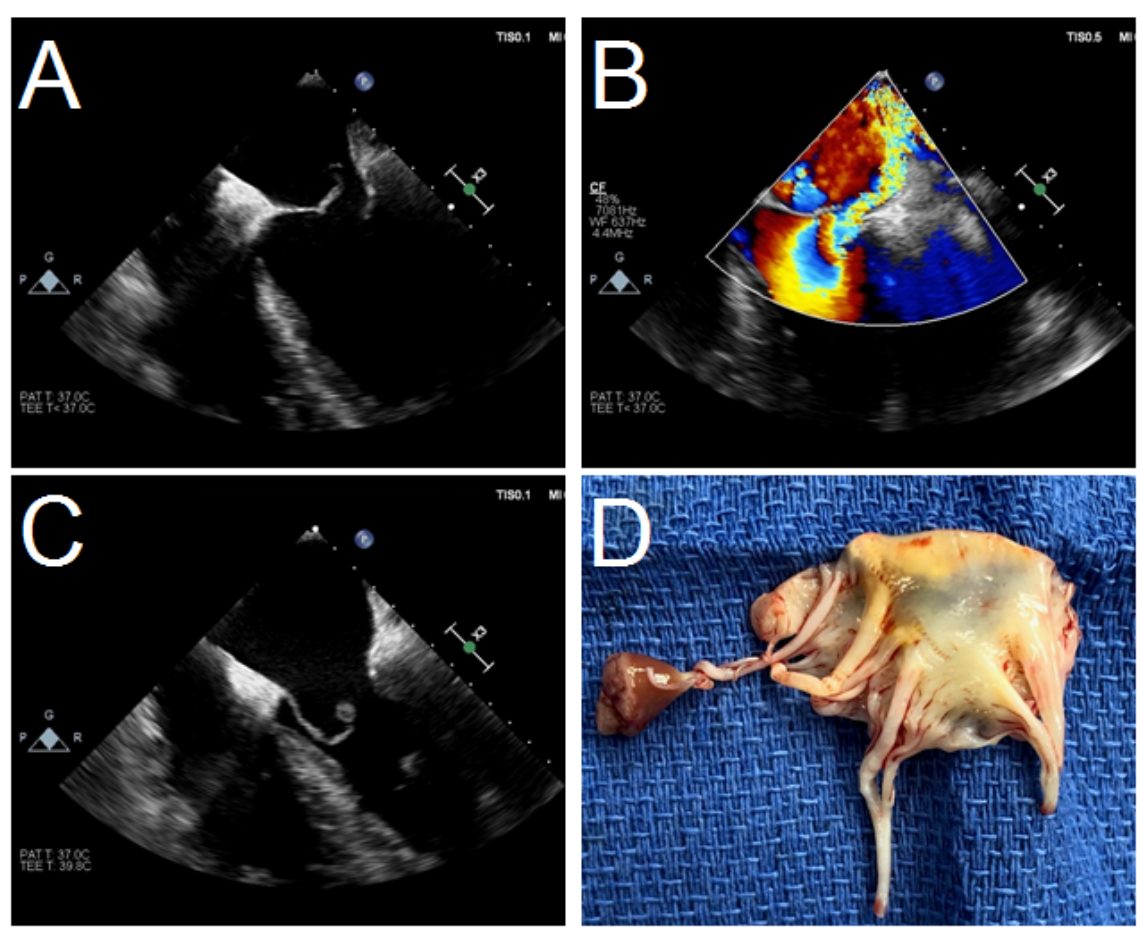

